\title{
Strong Correspondence of the Vaginal and Rectal Load of Group B Streptococci in Pregnant Women
}

\author{
Nabil Abdullah El Aila ${ }^{\mathrm{a}}$, Piet Cools ${ }^{\mathrm{b}}$, Pieter Deschaght ${ }^{\mathrm{b}}$, Guido Lopes dos Santos Santiago ${ }^{\mathrm{b}}$, \\ Geert Claeys $^{\mathrm{c}}$, Mario Vaneechoutte $\mathrm{b}^{\mathrm{b}, \mathrm{d}}$
}

\begin{abstract}
Background: To establish the potential role of the rectum as a reservoir for vaginal Streptococcus agalactiae (group B streptococci, GBS), both sites were sampled simultaneously and the bacterial loads of GBS were established by quantitative PCR (qPCR), for 100 pregnant women at $35-37$ weeks of gestation.
\end{abstract}

Methods: Vaginal and rectal occurrence of GBS was detected by qPCR, for 100 pregnant women at 35 - 37 weeks of gestation. DNA was extracted from vaginal $(V)$, rectal $(R)$ and vaginorectal $(V R)$ swabs using the NucliSENS EasyMAG platform and $c f b$-qPCR, specific for GBS, was carried out for the quantification of GBS.

Results: Ten women were found positive for both R and V swabs, 7 positive for $\mathrm{R}$ swabs only and 4 positive for $\mathrm{V}$ swabs only. Significant positive correlation was found between vaginal and rectal loads of GBS $(\mathrm{r}=0.701, \mathrm{P}<0.0001)$. In addition, the bacterial load of the 28 positive VR swabs (mean $\log 10$ cells/mL: 6.24, SD: 0.99; median: 5.82) was always higher than that of the V (mean: 5.72 , SD: 0.87; median: 5.56) or R swabs (mean: 5.52, SD 0.72; median: 5.16) separately $(\mathrm{P}<0.05)$.

Conclusions: Using qPCR, significant correlation between quantities of vaginal and rectal GBS loads was established. Comparison of sampling methods indicates that VR swabbing yields higher GBS loads and 7 more positive women than separate $\mathrm{V}$ and $\mathrm{R}$ swabbing, and therefore is preferable for sample collection for GBS detection in pregnant women.

Keywords: Streptococcus agalactiae; Vaginorectal swab; Bacterial

Manuscript accepted for publication August 26, 2013

\footnotetext{
${ }^{a}$ Medical Technology Department, Al-Aqsa University, Gaza, Palestine ${ }^{b}$ Laboratory Bacteriology Research, Department of Clinical Chemistry, Microbiology and Immunology, Ghent University, Belgium

${ }^{c}$ Laboratory for Microbiology, Ghent University Hospital, Ghent University, Belgium

${ }^{\mathrm{d}}$ Corresponding author: Mario Vaneechoutte, Laboratory Bacteriology Research, 3BlokA, 185 De Pintelaan, 9000 Ghent, Flanders. Belgium. Email: Mario.Vaneechoutte@UGent.be
}

doi: http://dx.doi.org/10.4021/jcgo185e load; Pregnant women; qPCR

\section{Introduction}

Group B Streptococcus (GBS, Streptococcus agalactiae) was recognized as the major cause of neonatal sepsis and meningitis in the 1970s [1]. Neonatal sepsis usually develops within 3 days after birth [2]. The maternal genital tract is the main source of neonatal infection [3] and GBS is transmitted vertically during labor and delivery in up to $80 \%$ of neonates born to colonized mothers [4]. Maternal streptococcal colonization is associated with increased risk of urinary tract infection and adverse pregnancy outcome, such as endometritis [5], chorioamnionitis [5,6], premature delivery and intrauterine death [7]. Sexual transmission may play a role in GBS colonization of the genital tract, because $45-91 \%$ of male partners of GBS-positive women are colonized with the identical serotype and/or strain $[8,9]$. The incidence of invasive neonatal GBS infection is reported to range from 0.5 to 3.0 per 1,000 live births, with $4-10 \%$ mortality associated with early-onset infections $[10,11]$.

Guidelines from the Centers for Disease Control and Prevention (CDC) recommend that all women should be screened at $35-37$ weeks of gestation and that those women found to be colonized with group B streptococci should receive intrapartum intravenous antibiotic prophylaxis either with penicillin $\mathrm{G}$ or with ampicillin [10], a treatment that has been shown to be effective in reducing the incidence of early-onset neonatal GBS infections [3].

The vaginal/rectal colonization rate of pregnant women ranges between $6.5 \%$ [12] and $43.6 \%$ [13] and may vary with characteristics such as age, parity, socio-economic status, geographic location [14], presence of sexually transmitted diseases [15] and sexual behavior [16]. Differences in colonization rates can also be attributed to variation in the culture and detection methods employed, including the media selected and collection sites used [17-19]. The aim of this study was to quantify group B streptococci in the vagina and rectum and to determine whether GBS loads in both sites corresponded, as an indication of the rectum as a reservoir of 
vaginal GBS. By comparing the GBS load in rectal, vaginal and rectovaginal swabs we also aimed to determine the best sampling method used for screening of group B streptococci from pregnant women.

\section{Materials and Methods}

\section{Study design}

One-hundred consecutive women between 35 and 37 weeks of gestation, attending the prenatal clinic at Ghent University Hospital (with an average of 1,200 deliveries per year) were enrolled in the study from June 2009 to January 2010. The study was approved by the Ethics Committee (IRB protocol nr 2007/096) of the Ghent University Hospital. All women provided informed consent prior to collection of samples.

\section{Collection and culture of specimens}

Rectovaginal, vaginal and rectal samples were collected using nylon flocked swabs that were submerged into $1 \mathrm{~mL}$ of ESwab transport medium (ESwab, Copan Diagnostics, Brescia, Italy).

Rectovaginal sampling was carried out by rotating an ESwab against the vaginal wall at the midportion of the vault. Subsequently, the swab was carefully withdrawn to prevent contamination with microflora from the vulva and introitus and the swab was inserted 1.5 to $2 \mathrm{~cm}$ beyond the anal sphincter and gently rotated to touch the anal crypts. Next, vaginal sampling was carried out by inserting the ESwab following the same procedure described above for swabbing the vaginal wall. Finally, an ESwab was used for rectal sampling as described above for the anal procedure of the rectovaginal sampling.

All samples were collected by midwives and transported to the Laboratory Bacteriology Research within 4 hours. Volumes of $200 \mu \mathrm{L}$ from the ESwab transport medium of the rectovaginal ESwabs were inoculated into separate tubes with $5 \mathrm{~mL}$ of Todd-Hewitt broth with $1 \%$ yeast extract, 15 $\mu \mathrm{g} / \mathrm{mL}$ nalidixic acid and $10 \mu \mathrm{g}$ colistin/mL (Lim broth, Becton Dickinson), which were incubated overnight aerobically at $37^{\circ} \mathrm{C}$.

\section{DNA extraction}

For DNA extraction, volumes of $200 \mu \mathrm{L}$ were taken from the ESwab transport medium of the vaginal, rectal and rectovaginal sample and another $200 \mu \mathrm{L}$ were taken from the incubated Lim broth tube. DNA extractions from the sample and from the Lim broth were performed using the NucliSENS easyMAG platform (BioMerieux), according to the manufacturer's instructions [20]. Briefly, $200 \mu \mathrm{L}$ of the Eswab transport medium from the rectovaginal ESwab or 200 $\mu \mathrm{L}$ from the incubated Lim broth was added to $1,800 \mu \mathrm{L}$ easyMAG lysis buffer and incubated for $10 \mathrm{~min}$ at room temperature and stored at $-80{ }^{\circ} \mathrm{C}$ until extraction. Nucleic acids were eluted in $100 \mu \mathrm{L}$ of elution buffer.

\section{qPCR assays}

GBS nucleic acid detection was based on targeting the $c f b$ gene, which encodes the CAMP factor. Primers and fluorescently labeled adjacent hybridization probes, namely, STB-F and STB-C, for detection of the $c f b$ gene were as described previously [21]. The qPCR was performed in a $20 \mu \mathrm{L}$ reaction volume on the LightCycler ${ }^{\circledR}$ v1.2 instrument using the LightCycler $^{\circledR}$ FastStart DNA Master HybProbe kit (Roche Diagnostics). Each reaction contained reagents to final concentrations of $0.4 \mu \mathrm{M}$ of each primer and $0.2 \mu \mathrm{M}$ of each hybridization probe and $5 \mu \mathrm{L}$ of DNA-extract. Thermal cycling parameters consisted of $10 \mathrm{~min}$ at $95{ }^{\circ} \mathrm{C}$ for denaturation and activation of the Taq polymerase, followed by 45 cycles of $10 \mathrm{sec}$ denaturation at $95^{\circ} \mathrm{C}, 10 \mathrm{sec}$ annealing at $55^{\circ} \mathrm{C}$ and $5 \mathrm{sec}$ extension at $72^{\circ} \mathrm{C}$.

Tenfold serial dilutions of a DNA extract of the type strain LMG $14694^{\mathrm{T}}$ were used to produce a standard curve. Positive controls (highly purified DNA from $5 \times 10^{8} \mathrm{GBS}$ cells) and negative controls (HPLC purified water) were included in each run. Samples that were positive by qPCR but negative by standard culture were investigated further by DNA sequence analysis of the amplification product.

\section{Statistical methods}

Data were analyzed using the Chi-square test and MannWhitney U-test with $p$ values of $<0.05$ considered as significant. Correlation of the bacterial load of GBS between the vagina and rectum was determined by the Spearman (rank) test and reported as Spearman's rho value (r).

\section{Results}

\section{Comparison of the sampling techniques using qPCR}

For a total of 100 women tested, 33 vaginorectal swabs were positive by qPCR after Lim Broth enrichment. Of these, 28 were positive by qPCR in at least one of the three samples ( $\mathrm{R}, \mathrm{V}$ or VR) without enrichment. Of these, all, 17 and 14 were positive by qPCR vaginorectally, rectally and vaginally respectively (Table 1).

After comparison of the bacterial load of GBS of each of the three sampling methods used, the bacterial load of the vaginorectal swab, as determined by qPCR, was always higher than that of the vaginal or rectal swabs separately $(\mathrm{P}$ $<0.05$ ) (Fig. 1, Table 1). The mean bacterial load (as determined by qPCR) of the VR swab was $6.24(\mathrm{SD}=0.99) \operatorname{logs}$, 


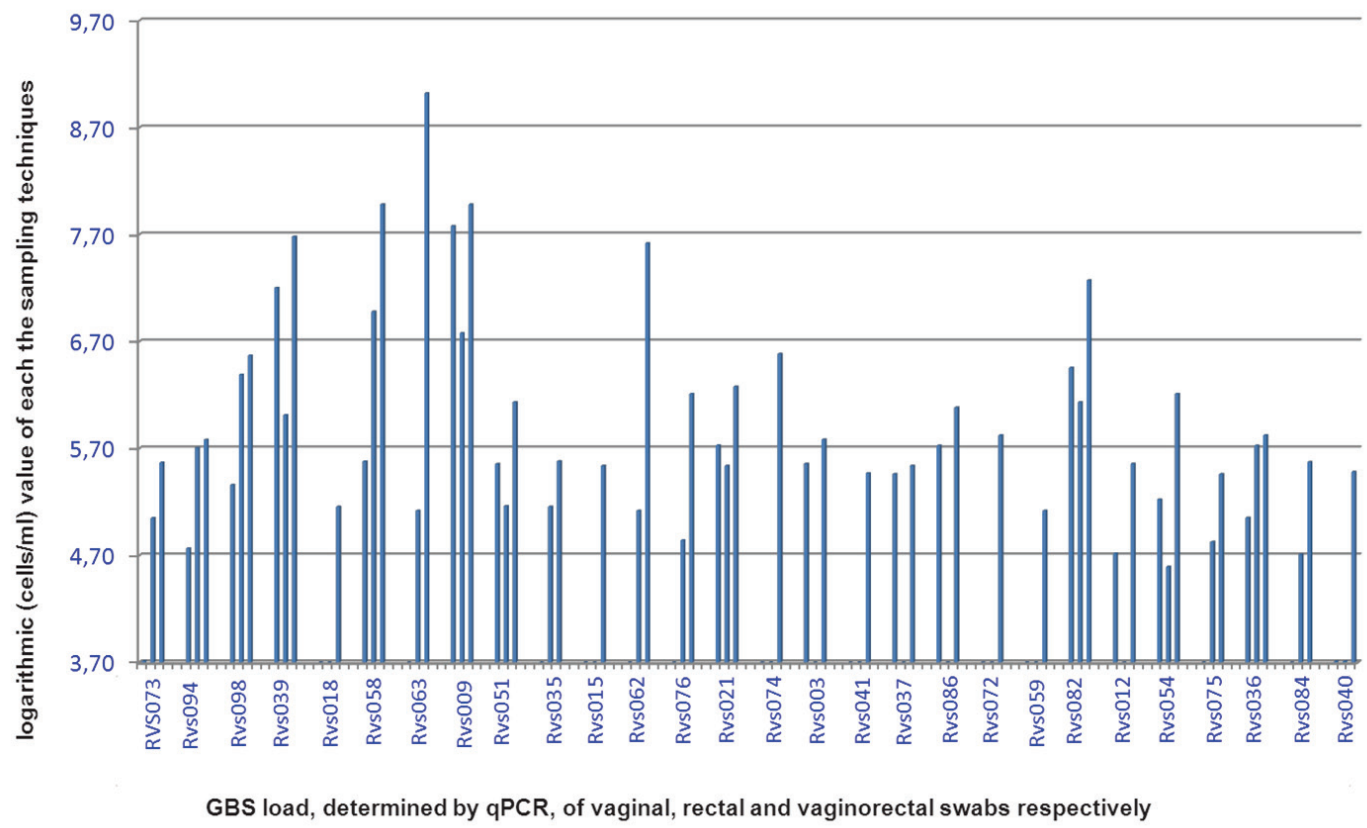

Figure 1. GBS bacterial load, determined by qPCR, for the vaginal, rectal and vaginorectal swabs respectively, for each woman positive for at least one swab. RVS: Rectal vaginal study.

namely, $1.66(\mathrm{SD}=1.26) \operatorname{logs}$ higher than the bacterial load of the vaginal swab and $1.50(\mathrm{SD}=0.97) \operatorname{logs}$ higher than that of the rectal swab.

\section{Bacterial loads of group B streptococci in the vagina and the rectum}

For the 10 women for which both vaginal and rectal samples were GBS qPCR positive, the mean difference between vaginal and rectal bacterial load of GBS was $0.77(\mathrm{SD}=0.39)$ logs. As shown in Table 1 and Figure 2, strong significant positive correlation was found between vaginal and rectal loads of GBS $(\mathrm{r}=0.701, \mathrm{P}<0.0001)$.

\section{Discussion}

\section{Background}

Several bacterial species are known to colonize both the gastrointestinal and the reproductive tract, and it is largely agreed that the rectum plays an important role as a source or reservoir for organisms that colonize the vagina.

Hallen et al [22] reported simultaneous occurrence of Mobiluncus spp. in the vagina and rectum. Holst et al [23] reported that the reservoirs of Mobilincus spp., Gardnerella vaginalis and Mycoplasma hominis are to be located in the intestinal tract. The rectum seems to be also the primary habitat of Candida spp. in patients with candidal vulvovaginitis [24]. We previously showed a high correlation between vaginal and rectal microflora, not only with regard to species composition but also with regard to the presence of genotypically identical strains [25].

Several culture-based studies have reported higher carriage rates of GBS in the rectum than in the vagina $[18,26$, 27], supporting the idea that the gastrointestinal tract is the reservoir for vaginal GBS. Meyn et al [28] showed that rectal colonization with GBS was the most significant predictor of vaginal colonization, suggesting that the vagina becomes colonized with GBS as a result of transfer of the organism from the rectum to the vagina. Our previous culture-based genotyping study showed a high correspondence of vaginal and rectal colonization with GBS, also at the strain level [25], because a total of 19 women was colonized by GBS in both rectum and vagina and moreover, 18 of these women had at least one vaginal and one rectal isolate with the same genotype.

To our knowledge, the present study is the first to quantify the inoculum of GBS in both rectum and vagina simultaneously and our results indicate a clear quantitative correspondence between bacterial loads present in the vagina and rectum, for group B streptococci. This is in correspondence with our previous quantitative, qPCR based, study, where we have shown significant correlation between quantities of vaginal and rectal lactobacilli and of Atopobium vaginae [29]. 
Table 1. Vaginal, Rectal and Vaginorectal Loads ( $\log 10 \mathrm{cells} / \mathrm{mL}$ ) of Group B Streptococci, as Determined by qPCR Without Lim Broth Enrichment, for the 33 Women for Which the Vaginorectal qPCR was Positive After Lim Broth Enrichment

\begin{tabular}{|c|c|c|c|c|}
\hline & $\begin{array}{l}\text { Subject study } \\
\text { designation }\end{array}$ & $\begin{array}{l}\text { Vaginal } \\
\text { load }\end{array}$ & Rectal load & Vaginorectal load \\
\hline 1 & RVSE009 & 7.78 & 6.78 & 7.98 \\
\hline 2 & RVSE021 & 5.73 & 5.54 & 6.28 \\
\hline 3 & RVSE036 & 5.05 & 5.73 & 5.82 \\
\hline 4 & RVSE039 & 7.20 & 6.01 & 7.68 \\
\hline 5 & RVSE051 & 5.56 & 5.16 & 6.13 \\
\hline 6 & RVSE054 & 5.22 & 4.59 & 6.21 \\
\hline 7 & RVSE058 & 5.58 & 6.98 & 7.98 \\
\hline 8 & RVSE082 & 6.46 & 6.13 & 7.27 \\
\hline 9 & RVSE094 & 4.77 & 5.71 & 5.78 \\
\hline 10 & RVSE098 & 5.36 & 6.39 & 6.57 \\
\hline 11 & RVSE003 & 5.56 & - & 5.78 \\
\hline 12 & RVSE012 & 4.72 & - & 5.56 \\
\hline 13 & RVSE037 & 5.46 & - & 5.54 \\
\hline 14 & RVSE086 & 5.73 & - & 6.08 \\
\hline 15 & RVSE035 & - & 5.16 & 5.58 \\
\hline 16 & RVSE062 & - & 5.12 & 7.62 \\
\hline 17 & RVSE063 & - & 5.12 & 9.02 \\
\hline 18 & RVSE073 & - & 5.05 & 5.57 \\
\hline 19 & RVSE075 & - & 4.83 & 5.46 \\
\hline 20 & RVSE076 & - & 4.84 & 6.21 \\
\hline 21 & RVSE084 & - & 4.71 & 5.57 \\
\hline 22 & RVSE015 & - & - & 5.54 \\
\hline 23 & RVSE018 & - & - & 5.16 \\
\hline 24 & RVSE040 & - & - & 5.48 \\
\hline 25 & RVSE041 & - & - & 5.47 \\
\hline 26 & RVSE059 & - & - & 5.12 \\
\hline 27 & RVSE072 & - & - & 5.82 \\
\hline 28 & RVSE074 & - & - & 6.59 \\
\hline 29 & RVSE008 & - & - & - \\
\hline 30 & RVSE010 & - & - & - \\
\hline 31 & RVSE014 & - & - & - \\
\hline 32 & RVSE083 & - & - & - \\
\hline \multirow[t]{2}{*}{33} & RVSE096 & - & - & - \\
\hline & Total positives & 14 & 17 & 28 \\
\hline
\end{tabular}




\section{Log 10 concentration of Group B streptococci in the vagina versus the} rectum

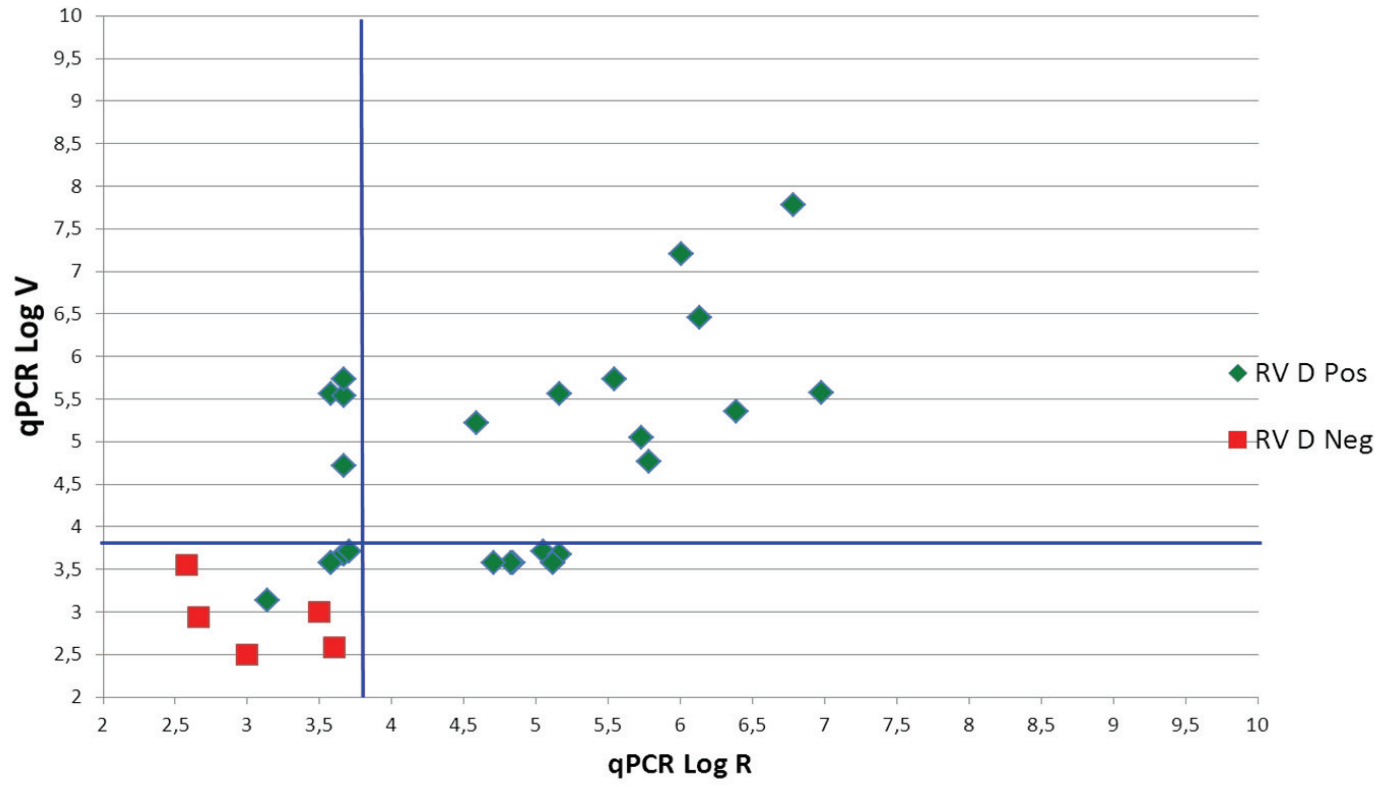

Figure 2. Log 10 (cells $/ \mathrm{mL}$ ) concentration of Group B streptococci in the vagina $(\mathrm{V})$ versus the rectum (R). RV D pos: vaginorectal swab is positive for GBS by means of qPCR; RV D neg: vaginorectal swab is negative for GBS by means of qPCR. Blue lines indicate $\mathrm{Cq}$ cut off values. Samples with values below these lines are considered qPCR negative. For these samples, reliable quantification was not possible and values indicated are presumptive. Samples with identical values are represented by only one dot.

\section{The importance of the sampling site for the sensitive de- tection of GBS}

Rectovaginal swabs have been reported to provide high bacterial yields $[10,25,30-32]$ and have therefore been recommended for GBS screening [10]. However, Nomura et al [33] found no significant difference in detection rates between vaginal and rectal samples and Gupta and Briski [34] reported a similar detection rate of $23.8 \%$ of GBS when using rectovaginal and vaginal sampling. Votava et al [32] even found that the GBS detection rate using rectovaginal samples was only $16.9 \%$, whereas the use of separate vaginal and rectal swabs yielded 22.7 and $24.1 \%$ GBS positive women, respectively. Also, several obstetric departments still use vaginal sampling only to assess GBS positivity.

In a previous study, we showed that, to maximize GBS carriage detection rates, both the anatomic site of sampling and the culture methods used are important [18]. In that study, we compared three sampling techniques, namely rectovaginal swabbing, vaginal swabbing only and rectal swabbing only, using the Eswab, and we concluded that for culture, rectovaginal sampling increased the number of GBS positive women detected, compared to vaginal and/or rectal sampling, namely the number of GBS positive women on the basis of rectovaginal swabbing $(n=22)$ was significantly higher than the number of GBS positive women on the basis of vaginal sampling $(n=11)$, and higher than the number on the basis of rectal sampling $(n=18)$ [18].

Because of the abovementioned conflicting results, it was of interest to compare the three sampling methods with respect to their sensitivity for qPCR-based GBS detection.

Our present qPCR-based results, whereby 17 women were positive for the rectum and 14 for the vagina, correspond with previous reports that GBS colonization of rectal samples is $18 \%$ to $24 \%$ higher than that of vaginal samples $[18,35,36]$. Our present data which indicate that overall positivity of rectovaginal sampling is highest (28 positives) and that all women with rectal or vaginal samples positive, were also positive for the rectovaginal samples, are in correspondence with those of other studies that find rectovaginal sampling more appropriate than vaginal sampling only [1, $10,22]$. For example, in an analysis of 651 specimens, the combination of separate rectal and vaginal sampling enabled detection of $97.8 \%$ of GBS carriers, compared to $31.8 \%$ of positives as established by vaginal sampling only [37].

\section{Declaration of Interest}

The authors report no conflicts of interest. 


\section{References}

1. Allen UD, Navas L, King SM. Effectiveness of intrapartum penicillin prophylaxis in preventing early-onset group B streptococcal infection: results of a meta-analysis. CMAJ. 1993;149(11):1659-1665.

2. Bergeron MG, Ke D, Menard C, Picard FJ, Gagnon M, Bernier M, Ouellette M, et al. Rapid detection of group $\mathrm{B}$ streptococci in pregnant women at delivery. N Engl J Med. 2000;343(3):175-179.

3. Boyer KM, Gotoff SP. Strategies for chemoprophylaxis of GBS early-onset infections. Antibiot Chemother. 1985;35:267-280.

4. Schuchat A. Group B streptococcal disease in newborns: a global perspective on prevention. Biomed Pharmacother. 1995;49(1):19-25.

5. Winn HN. Group B Streptococcus infection in pregnancy. Clin Perinatol. 2007;34(3):387-392.

6. Yancey MK, Duff P, Clark P, Kurtzer T, Frentzen BH, Kubilis P. Peripartum infection associated with vaginal group B streptococcal colonization. Obstet Gynecol. 1994;84(5):816-819.

7. Tyrrell GJ, Senzilet LD, Spika JS, Kertesz DA, Alagaratnam M, Lovgren M, Talbot JA. Invasive disease due to group B streptococcal infection in adults: results from a Canadian, population-based, active laboratory surveillance study--1996. Sentinel Health Unit Surveillance System Site Coordinators. J Infect Dis. 2000;182(1):168173.

8. Franciosi RA, Knostman JD, Zimmerman RA. Group B streptococcal neonatal and infant infections. J Pediatr. 1973;82(4):707-718.

9. Yamamoto T, Nagasawa I, Nojima M, Yoshida K, Kuwabara Y. Sexual transmission and reinfection of group B streptococci between spouses. J Obstet Gynaecol Res. 1999;25(3):215-219.

10. Verani JR, McGee L, Schrag SJ. Prevention of perinatal group B streptococcal disease--revised guidelines from CDC, 2010. MMWR Recomm Rep. 2010;59(RR-10):136.

11. Schrag SJ, Whitney CG, Schuchat A. Neonatal group B streptococcal disease: how infection control teams can contribute to prevention efforts. Infect Control Hosp Epidemiol. 2000;21(7):473-483.

12. Yucesoy G, Caliskan E, Karadenizli A, Corakci A, Yucesoy I, Huseyinoglu N, Babaoglu K. Maternal colonisation with group B Streptococcus and effectiveness of a culture-based protocol to prevent early-onset neonatal sepsis. Int J Clin Pract. 2004;58(8):735-739.

13. Gavino M, Wang E. A comparison of a new rapid realtime polymerase chain reaction system to traditional culture in determining group B Streptococcus colonization. Am J Obstet Gynecol. 2007;197(4):388 e381-384.

14. Regan JA, Klebanoff MA, Nugent RP. The epidemiol- ogy of group B streptococcal colonization in pregnancy. Vaginal Infections and Prematurity Study Group. Obstet Gynecol. 1991;77(4):604-610.

15. Persson K, Bjerre B, Hansson H, Forsgren A. Several factors influencing the colonization of group B streptococci--rectum probably the main reservoir. Scand J Infect Dis. 1981;13(3):171-175.

16. Foxman B, Gillespie BW, Manning SD, Marrs CF. Risk factors for group B streptococcal colonization: potential for different transmission systems by capsular type. Ann Epidemiol. 2007;17(11):854-862.

17. Davies HD, Adair CE, Partlow ES, Sauve R, Low DE, McGeer A. Two-year survey of Alberta laboratories processing of antenatal group B streptococcal (GBS) screening specimens: implications for GBS screening programs. Diagn Microbiol Infect Dis. 1999;35(3):169176.

18. El Aila NA, Tency I, Claeys G, Saerens B, Cools P, Verstraelen $\mathrm{H}$, Temmerman M, et al. Comparison of different sampling techniques and of different culture methods for detection of group B Streptococcus carriage in pregnant women. BMC Infect Dis. 2010;10:285.

19. El Aila NA, Tency I, Claeys G, Verstraelen H, Deschaght P, Decat E, Lopes dos Santos Santiago G, et al. Comparison of culture with two different qPCR assays for detection of rectovaginal carriage of Streptococcus agalactiae (group B streptococci) in pregnant women. Res Microbiol. 2011;162(5):499-505.

20. Dundas N, Leos NK, Mitui M, Revell P, Rogers BB. Comparison of automated nucleic acid extraction methods with manual extraction. J Mol Diagn. 2008;10(4):311-316.

21. Ke D, Menard C, Picard FJ, Boissinot M, Ouellette M, Roy PH, Bergeron MG. Development of conventional and real-time PCR assays for the rapid detection of group B streptococci. Clin Chem. 2000;46(3):324-331.

22. Hallen A, Pahlson C, Forsum U. Rectal occurrence of Mobiluncus species. Genitourin Med. 1988;64(4):273275.

23. Holst E. Reservoir of four organisms associated with bacterial vaginosis suggests lack of sexual transmission. J Clin Microbiol. 1990;28(9):2035-2039.

24. Miles MR, Olsen L, Rogers A. Recurrent vaginal candidiasis. Importance of an intestinal reservoir. JAMA. 1977;238(17):1836-1837.

25. El Aila NA, Tency I, Claeys G, Saerens B, De Backer E, Temmerman M, Verhelst R, et al. Genotyping of Streptococcus agalactiae (group B streptococci) isolated from vaginal and rectal swabs of women at 35-37 weeks of pregnancy. BMC Infect Dis. 2009;9:153.

26. Boyer KM, Gadzala CA, Kelly PD, Gotoff SP. Selective intrapartum chemoprophylaxis of neonatal group B streptococcal early-onset disease. III. Interruption of mother-to-infant transmission. J Infect Dis. 
1983;148(5):810-816.

27. Dillon HC, Jr., Gray E, Pass MA, Gray BM. Anorectal and vaginal carriage of group B streptococci during pregnancy. J Infect Dis. 1982;145(6):794-799.

28. Meyn LA, Krohn MA, Hillier SL. Rectal colonization by group B Streptococcus as a predictor of vaginal colonization. Am J Obstet Gynecol. 2009;201(1):76 e71-77.

29. El Aila NA, Tency I, Saerens B, De Backer E, Cools P, dos Santos Santiago GL, Verstraelen H, et al. Strong correspondence in bacterial loads between the vagina and rectum of pregnant women. Res Microbiol. 2011;162(5):506-513.

30. Dunne WM, Jr. Comparison of selective broth medium plus neomycin-nalidixic acid agar and selective broth medium plus Columbia colistin-nalidixic acid agar for detection of group B streptococcal colonization in women. J Clin Microbiol. 1999;37(11):3705-3706.

31. Philipson EH, Palermino DA, Robinson A. Enhanced antenatal detection of group B Streptococcus colonization. Obstet Gynecol. 1995;85(3):437-439.

32. Votava M, Tejkalova M, Drabkova M, Unzeitig V, Braveny I. Use of GBS media for rapid detection of group B streptococci in vaginal and rectal swabs from women in labor. Eur J Clin Microbiol Infect Dis. 2001;20(2):120122.

33. Nomura ML, Passini Junior R, Oliveira UM. Selective versus non-selective culture medium for group B Streptococcus detection in pregnancies complicated by preterm labor or preterm-premature rupture of membranes. Braz J Infect Dis. 2006;10(4):247-250.

34. Gupta C, Briski LE. Comparison of two culture media and three sampling techniques for sensitive and rapid screening of vaginal colonization by group B Streptococcus in pregnant women. J Clin Microbiol. 2004;42(9):3975-3977.

35. Madani TA, Harding GK, Helewa M, Alfa MJ. Screening pregnant women for group B streptococcal colonization. Infection. 1998;26(5):288-291.

36. Quinlan JD, Hill DA, Maxwell BD, Boone S, Hoover F, Lense JJ. The necessity of both anorectal and vaginal cultures for group B Streptococcus screening during pregnancy. J Fam Pract. 2000;49(5):447-448.

37. Platt MW, McLaughlin JC, Gilson GJ, Wellhoner MF, Nims LJ. Increased recovery of group B Streptococcus by the inclusion of rectal culturing and enrichment. Diagn Microbiol Infect Dis. 1995;21(2):65-68. 